\title{
Leptomeningeal form of Immunoglobulin G4-related hypertrophic meningitis with perivascular spread: a case report and review of the literature
}

\author{
Jasmina Boban ${ }^{1} \cdot$ Selin Ardalı ${ }^{2}$ Majda M. Thurnher $^{3}$
}

Received: 4 April 2018 / Accepted: 24 April 2018 / Published online: 7 May 2018

(C) The Author(s) 2018

\begin{abstract}
Purpose Immunoglobulin G4 (IgG4)-related disease represents a spectrum of fibro-inflammatory disorders that affects various organ systems, including the central nervous system.

Methods Here we present the case of lgG4-related hypertrophic meningitis with exclusively leptomeningeal involvement and spread via perivascular spaces.

Results A 58-year-old male patient presented with complex partial seizures. Initial computed tomography examination showed left frontal sulcal hyperdensity. Subsequent magnetic resonance examination revealed FLAIR hyperintensity in the central sulcus, with post-contrast enhancement in the form of "dotted line." Physical examination, routine laboratory, and cerebrospinal fluid analyses were unremarkable. Meningeal biopsy confirmed IgG4-related meningitis. After corticosteroid treatment, a complete resolution of imaging findings was observed. Two months later, the patient presented with relapsing neurological symptoms and radiological findings in postcentral, precentral, and temporal sulci, resembling the form of "dotted line" contrast enhancement. In addition, linear intraparenchymal enhancement that followed perivascular spaces was seen in the left parietal lobe. After repeated steroid therapy, all lesions resolved completely.

Conclusion We reported the first case of isolated IgG4-related leptomeningeal involvement with a "dotted line" enhancement and perivascular intraparenchymal spread. Although IgG4-related meningitis represents a rare disease, both clinicians and radiologists should include this condition in the differential diagnosis of unclear leptomeningeal disease.
\end{abstract}

Keywords Meningitis $\cdot \operatorname{IgG} 4 \cdot$ Magnetic resonance imaging (MRI)

\section{Abbreviations}

IgG4 Immunoglobulin G4

Majda M. Thurnher

majda.thurnher@meduniwien.ac.at

Jasmina Boban

jasmina.boban@mf.uns.ac.rs

Selin Ardal1

selinardali@gmail.com

1 Faculty of Medicine, Department for Radiology, University of Novi Sad, Hajduk Veljkova 3, Novi Sad 21000, Serbia

2 Department of Radiology, Hacettepe University Faculty of Medicine, Sihhiye, 06100 Ankara, Turkey

3 Section of Neuroradiology and Musculoskeletal Radiology, Department for Biomedical Imaging and Image-guided Therapy, Medical University of Vienna, Waehringer Guertel 18-20, 1090 Vienna, Austria
FLAIR Fluid attenuation inversion recovery

CNS Central nervous system

IHCPM Idiopathic hypertrophic cranial pachymeningitis

CSF Cerebrospinal fluid

CT Computed tomography

MR Magnetic resonance

T1W T1-weighted

T2W T2-weighted

\section{Introduction}

Immunoglobulin G4 (IgG4)-related disease encompasses a spectrum of fibro-inflammatory disorders characterized by IgG4-positive plasma cell infiltration that can affect almost every organ system [1]. The disease was first described in the pancreas, and then, in the salivary and lacrimal glands, thyroid, kidney, bile ducts, lungs, and retroperitoneum [1, 2]. 
Histopathologic features include lymphoplasmacytic infiltration of IgG4+ plasma cells, storiform fibrosis, and obliterative phlebitis. Central nervous system (CNS) involvement is relatively rare and mostly seen as hypophysitis. Hypertrophic pachymeningitis is a focal or diffuse thickening of the intracranial and/or spinal meninges and is a recently recognized part of the IgG4-related disease spectrum [3, 4]. Hypertrophic pachymeningitis can be observed in a wide range of diseases, including malignant, immunological, infectious, and vasculitic conditions, and can also be idiopathic. Many of the cases previously described as idiopathic hypertrophic cranial pachymeningitis (IHCPM) might actually belong to the IgG4-related disease spectrum [5].

To date, several cases of pachymeningitis - but only three cases of leptomeningeal involvement in IgG4-related disease have been described [6]. To the best of our knowledge, no case of an isolated leptomeningeal form of IgG4-related disease with perivascular spread has been described. Here, we present the case of histologically proven lgG4-related hypertrophic meningitis with exclusively leptomeningeal involvement and spread via the perivascular spaces.

\section{Case presentation}

A 58-year-old previously healthy male patient presented with complex partial seizures. Physical examination and routine laboratory tests were unremarkable. Cerebrospinal fluid (CSF) analysis showed a normal cell count, and normal protein and glucose levels. Initial computed tomography (CT) of the brain showed a subtle sulcal hyperdensity in the left frontal lobe (Fig. 1a). Brain magnetic resonance (MR) imaging revealed a fluid-attenuated inversion recovery (FLAIR) hyperintense left central sulcus (Fig. 1b). On post-contrast T1-weighted images, nodular leptomeningeal enhancement in the form of a "dotted line," corresponding to the sulcal FLAIR hyperintensity, was observed (Fig. 1c). The underlying gray matter of the central gyrus was FLAIR/T2-weighted hyperintense. On the follow-up MR examination, the enhancement in the central sulcus became thicker and more linear. At this point, a subtle linear enhancement along the perivascular spaces was detected in the underlying white matter of the parietal lobe. The patient underwent surgical biopsy that revealed meningeal deposits of collagenous fibrous tissue, imbued with thick reactive-proliferative, mostly inflammatory infiltrate with numerous plasma cells and monocytes, and several giant round cells. Inflammatory changes were observed along the small blood vessels also, with no signs of obliterative phlebothrombosis. Most plasma cells were positive for IgG4 antibodies on immunohistochemistry, thus indicating the diagnosis of IgG4-related meningitis. Serum IgG and IgG4 levels were within normal range. The workup for immunologic diseases and malignancy was negative. There were no relevant symptoms or findings compatible with the IgG4-related disease spectrum in any other organ systems. After corticosteroid treatment, there was a complete resolution of imaging findings. However, 2 months later, the patient presented with new neurological symptoms (headache, speech difficulties, sleeping disorder). MR revealed FLAIR hyperintensity in the left precentral and postcentral sulci and in the sulci of the left temporal lobe (Fig. 1f-g). The postcentral gyrus appeared hyperintense on FLAIR and T2W. "Dotted line" enhancement, similar to that observed in the initial lesion, was detected in the affected sulci (Fig. 1i, j). In addition, a more prominent, linear intraparenchymal enhancement that followed the perivascular spaces was clearly seen in the left parietal lobe (Fig. 1k, 1). New lesions were attributed to the relapse of idiopathic IgG4-related disease. After repeated administration of steroid therapy, lesions resolved almost completely (Fig. $1 \mathrm{~m}$ ). A discrete dotted micronodular enhancement was still evident in precentral sulcus on the left, as well as along perivascular spaces in the left hemisphere.

\section{Discussion and conclusions}

IgG4-related disease is a recently recognized spectrum of complex, immune-mediated, sclerosing inflammatory disorders with common pathologic, clinical, and serologic characteristics [1]. The condition was first described in the pancreas, and then, there were reports of this condition in the orbit, salivary glands, lymph nodes, lungs, kidneys, and other organs [2]. Hypertrophic pachymeningitis is a rare neurologic manifestation of the IgG4-related disease spectrum characterized by localized or diffuse thickening of the dura mater [3, 4]. Recently, IgG4-related pachymeningitis has been suggested to represent some of the cases that were previously diagnosed as idiopathic hypertrophic cranial pachymeningitis (IHCPM) [5].

IgG4-related pachymeningitis generally occurs in men during the fifth and sixth decades. Typical clinical symptoms include headache, seizures or focal deficits, compression or involvement of cranial nerves leading to neuropathies, vessel occlusions, and dural venous sinus occlusions [6]. On CT/MR imaging, hypertrophic pachymeningitis is characterized by diffuse or masslike thickening of the dura over the cerebral hemispheres and/or the spinal cord [7-9]. Some cases with bulging masses connected to the dura have also been described [9].

To date, to the best of our knowledge, no cases of isolated leptomeningeal IgG4-related meningitis with perivascular spread have been described.

In our patient, an isolated leptomeningeal involvement was present in the frontal, parietal, and temporal sulci. Leptomeningeal enhancement had an interesting pattern of a "dotted line," consisting of a small nodular thickening 

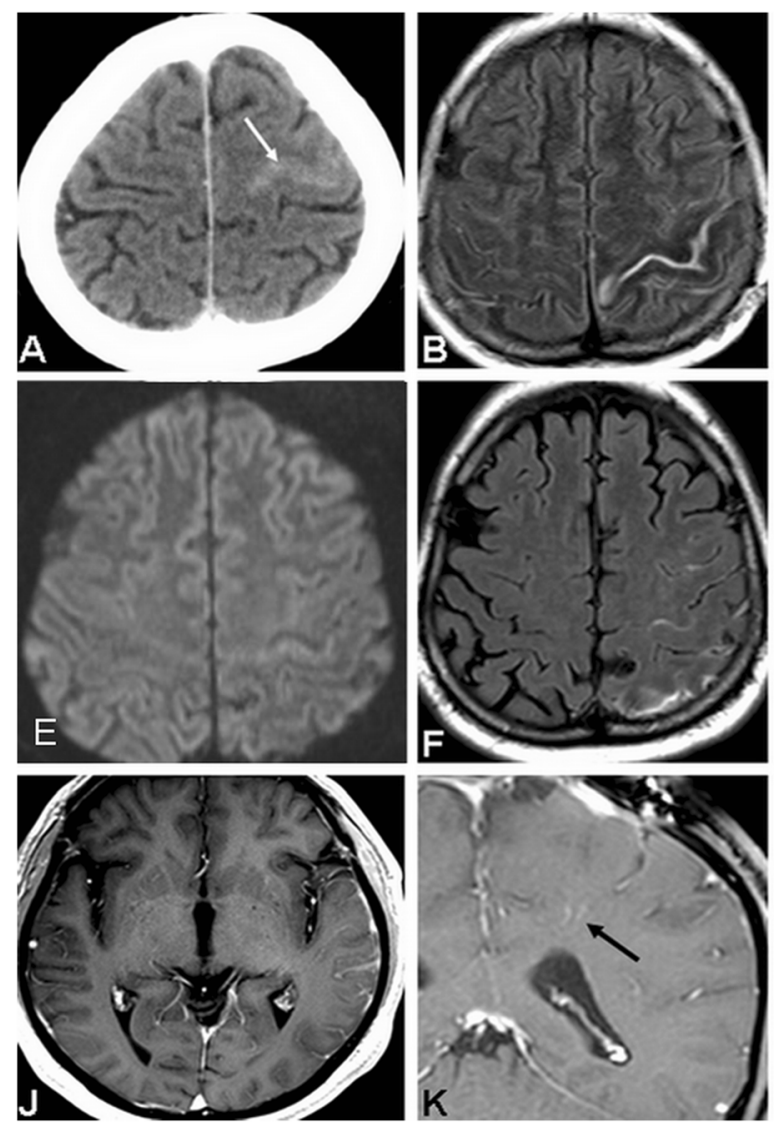

Fig. 1 a-m Axial pre-contrast CT scan showed slightly hyperdense central sulcus (a, white arrow). Sulcal hyperintensity was detected on axial FLAIR image (b, white arrow). After contrast administration (c, white arrow), a leptomeningeal pattern of "dotted line" enhancement was observed, while on native T1W images (d) the sulcal narrowing was evident only. There was no restricted diffusion observed (e). Relapse occurred after 2 months, affecting postcentral, precentral sulcus (f), with no

of the leptomeninges. Nodular leptomeningeal enhancement can also be observed in malignant or vasculitic diseases [10]. Also, leptomeningeal enhancement was described, although rarely, in some autoimmune disorders. In rheumatoid arthritis, a typical pattern of associated thick dural and leptomeningeal enhancement was observed [11]. U-shaped leptomeningeal enhancement that varied in size, number, and location over time was described in Susac's syndrome [12]. A recently described entity, Rosai-Dorfman disease, is an idiopathic histiocytic disorder with sinus histiocytosis and massive lymphadenopathy. Several cases with diffuse meningeal involvement on MRI were described, but more commonly in the form of diffuse dural enhancement, such as that in meningioma, lymphoma, or chronic inflammation [13].

Soon after the onset of the leptomeningeal disease, a subtle contrast enhancement was noted in the underlying white matter, along the perivascular spaces, representing inflammatory spread along the perivascular spaces. In addition, T2W/
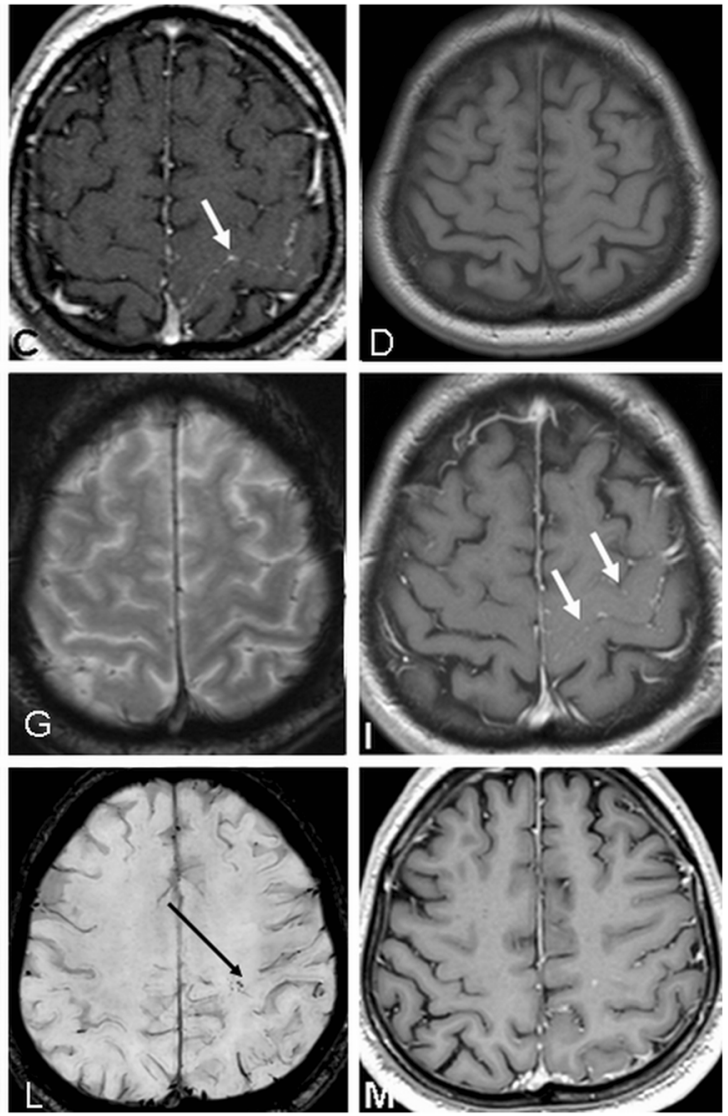

hemosiderin deposits on $\mathrm{T} 2 * \mathrm{~W}$ images (g) and the typical "dotted line" leptomeningeal enhancement (i, $\mathbf{j}$, white arrows). Spread along perivascular spaces was observed as linear contrast enhancement along dilated veins (k, $\mathbf{l}$, black arrows). After corticosteroid treatment, 6 weeks later, almost complete resolution was observed $(\mathbf{m})$. A discrete dotted micronodular enhancement was still evident in precentral sulcus on the left, as well as along perivascular spaces in the left hemisphere

FLAIR signal changes without contrast enhancement were evident in the underlying cortex. These changes probably represented vasogenic edema (no signs of restricted diffusion were present), due to venous congestion and deprived venous outflow. There were no signs of dural involvement or dural sinus thrombosis. Post-contrast T1W images showed enhancement in the narrowed sulci, as in previously described cases of dural thickening [7-9].

There have been only three cases of leptomeningeal involvement in IgG4-related disease reported in the literature $[6,9,14]$. However, in two patients, a history of concomitant rheumatoid arthritis was present; thus, a certain bias in the differential diagnosis was created. One of these two patients also had an adjacent diffuse pachymeningeal enhancement [9]. The third patient was described in a pathologic study that reviewed ten cases of unexplained meningeal pathologic findings, with no imaging or clinical data available. In five cases, after additional immunohistological analysis, IgG4-related disease was confirmed; leptomeningeal involvement was 
described in one of these cases. Histological findings were similar to those in our case, with a lymphoplasmacytic perivascular inflammatory infiltrate with less prominent sclerosis, and no signs of obliterative phlebitis [6].

Meningeal biopsy is the gold standard for establishing the diagnosis of IgG4-related disease [15]. Diagnostic criteria for IgG4-related pachymeningitis are based on three characteristic histological findings: lymphoplasmacytic infiltration of IgG4-positiveplasma cells, storiform fibrosis, and obliterative phlebitis. In our patient, there was a thick inflammatory infiltrate of plasmacytic and monocytic cells, with the majority of plasma cells positive for IgG4 antibodies. The inflammatory infiltrate was also present along the blood vessels, without signs of extensive fibrosis, concordant with the previous pathologic study [6]. There are no single immunohistochemical features that are pathognomonic for this disease. However, a cutoff value of $10 \operatorname{IgG} 4+$ plasma cells/HPF, and an IgG4+/IgG+ plasma cell ratio $>40 \%$, are recognized as sufficient for the diagnosis of IgG4-related disease of the meninges [15].

CSF analysis is performed to exclude other pathologies, such as CNS infections or malignant disease. Usually, the results of CSF analysis reveal normal glucose levels, normal or slightly elevated protein levels, and a variable degree of pleocytosis (lymphocytic, monocytic) [16]. Results from previous studies have implied moderate to severe damage to the blood-brain barrier, as in other forms of inflammatory meningitis. A higher elevation of CSF IgG4 levels in patients with IgG4-related pachymeningitis when compared with patients with infectious, neoplastic, and inflammatory meningitis has also been reported [16].

Serum IgG4 level may or may not be elevated [17]. In our patient, and in the majority of the cases described in the literature, serum IgG4 levels were within normal ranges.

Although currently there is no consensus about the treatment of IgG4-related pachymeningitis, glucocorticoid therapy is the first choice. Recurrent disease after corticosteroid therapy rarely occurs. However, in our case, recurrence was observed after cessation of steroid therapy after a short period of time, similar to the case described by Shapiro et al. [18]. Cases with recurrent disease are treated with the addition of immunosuppressive, steroid-sparing agents, such as azathioprine, mycophenolate mofetil, methotrexate, and rituximab [1].

In conclusion, this is the first case of isolated IgG4-related leptomeningeal involvement with a "dotted line" enhancement and perivascular intraparenchymal spread. Although a rare disease, both clinicians and radiologists should be aware of this condition as part of the differential diagnosis in patients with leptomeningeal disease. A prompt diagnosis with meningeal biopsy and a specific therapeutic approach might prevent long-term neurological complications.

\section{Compliance with ethical standards}

Funding The Medical University of Vienna funded Open Access.

Conflict of interest The authors declare that they have no conflict of interest.

Ethical approval All procedures performed in the studies involving human participants were in accordance with the ethical standards of the institutional and/or national research committee and with the 1964 Helsinki Declaration and its later amendments or comparable ethical standards. For this type of the study formal consent is not required.

Informed consent Informed consent was obtained from the patient's relative.

Open Access This article is distributed under the terms of the Creative Commons Attribution 4.0 International License (http:// creativecommons.org/licenses/by/4.0/), which permits unrestricted use, distribution, and reproduction in any medium, provided you give appropriate credit to the original author(s) and the source, provide a link to the Creative Commons license, and indicate if changes were made.

\section{References}

1. Stone JH, Zen Y, Deshpande V (2012) IgG4-related disease. N Engl J Med 366:539-551

2. Hamano H, Kawa S, Horiuchi A, Unno H, Furuya N, Akamatsu T, Fukushima M, Nikaido T, Nakayama K, Usuda N, Kiyosawa K (2001) High serum IgG4 concentrations in patients with sclerosing pancreatitis. N Engl J Med 344:732-738

3. Della-Torre E, Galli L, Franciotta D, Bozzolo EP, Briani C, Furlan R, Roveri L, Sessa M, Passerini G, Sabbadini MG (2014) Diagnostic value of IgG4 indices in IgG4-related hypertrophic pachymeningitis. J Neuroimmunol 266:82-86

4. De Virgilio A, de Vincentilis M, Inghilleri M et al (2016) Idiopathic hypetrophicpachymeningitis: an autoimmune IgG4-relateddisease. Immunol Res 65:386-394. https://doi.org/10.1007/s12026-0168863-1

5. Hassan KM, Prabal Deb, Bhatoe HS (2011) Idiopathic hypertrophic cranial pachymeningitis: three biopsy-proven cases including one case with abdominal pseudotumor and review of the literature. Ann Indian Acad Neurol 14: 189-193

6. Lindstrom KM, Cousar JB, Lopes MB (2010) IgG4-related meningeal disease: clinico-pathological features and proposal for diagnostic criteria. Acta Neuropathol 120:765-776

7. D'Andrea G, Trillò G, Celli P et al (2004) Idiopathic intracranial hypertrophic pachymeningitis: two case reports and review of the literature. Neurosurgery 27:199-204

8. Choi SH, Lee SH, Khang SK, Jeon SR (2010) IgG4-related sclerosing pachymeningitis causing spinal cord compression. Neurology 75:1388-1390

9. Mehta SH, Switzer J, Biddinger P, Rojiani AM (2014) IgG4-related leptomeningitis: a reversible cause of rapidly progressive cognitive decline. Neurology 82(6):540-542

10. Smirniotopoulos JG, Murphy FM, Rushing EJ, Rees JH, Schroeder JW (2007) Patterns of contrast enhancement in the brain and meninges. Radiographics 27(2):525-552

11. Servioli MJ, Chugh C, Lee JM, Biller J (2011) Rheumatoid meningitis. Front Neurol 2:84 
12. Engisch R, Titelbaum DS, Chilver-Stainer L, Kellner-Weldon (2016) Susac's syndrome: leptomeningeal enhancement on 3D FLAIR MRI. Mult Scler 22(7): 972-974

13. Joshi SS, Joshi S, Muzumdar G et al (2017) Cranio-spinal Rosai Dorfman disease: case series and literature review. Br J Neurosurg 22:1-8

14. Hiraga A, Ozaki D, Tsuneyama A, Ito S, Koide K, Kuwabara S (2015) Corticosterod-responsive leptomeningitis with IgG4positive plasma-cell infiltration. J Neurol Sci 357:338-340

15. Lu LX, Della-Torre E, Stone JH, Clark SW (2014) IgG4-related hypertrophic pachymeningitis: clinical features, diagnostic criteria, and treatment. JAMA Neurol 71(6):785-793
16. Della-Torre E, Passerini G, Furlan R et al (2013) Cerebrospinal fluid analysis in IgG4-related hypertrophic pachymeningitis. J Rheumatol 40(11):1927-1929

17. Divatia M, Kim SA, Ro JY (2012) IgG4-related sclerosing disease, an emerging entity: a review of a multi-system disease. Yonsei Med J 53:15-34

18. Shapiro KA, Bove RM, Volpicell ER et al (2012) Relapsing course of immunoglobulin G4-related pachymeningitis. Neurology 79: 604-606 